\title{
Fühlen(d) Lernen: Zur Sozialisation und Entwicklung von Emotionen im Kulturvergleich
}

Leberecht Funk ${ }^{1}$, Birgitt Röttger-Rössler ${ }^{1}$ und Gabriel Scheidecker ${ }^{1}$

(1)

Institut für Ethnologie, FUB, Habelschwerdter Allee 45, 14195 Berlin, Deutschland

Birgitt Röttger-Rössler

Email: Birgitt.Roettger-Roessler@fu-berlin.de

Online publiziert: 17. Oktober 2012

Zusammenfassung

Forschungen zur Sozialisation und Ontogenese von Emotionen sind in der Sozial- und Kulturanthropologie bisher weitestgehend vernachlässigt worden. Entwicklungspsychologen beschäftigen sich dagegen intensiv mit diesem Thema, wobei sich jedoch das Gros ihrer Studien auf euro-amerikanische Gesellschaften beschränkt und somit kaum Aussagen über interkulturelle Divergenzen zulässt. In diesem Artikel vergleichen wir die Sozialisation von Emotionen in zwei nicht-westlichen Gesellschaften: den Bara in Madagaskar und den Tao auf der taiwanesischen Insel Lanyu. Es wird aufgezeigt, wie folk models von Person, Emotion und Entwicklung mit den jeweiligen lokalen Erziehungspraktiken verknüpft sind. In beiden Gesellschaften werden von den Bezugspersonen Sanktionierungsstrategien angewendet, die mit einem hohen Maß an emotionaler Erregung einhergehen. Während bei den Bara „Furcht“ induziert wird, kommt es bei den Tao zu einer Evokation von „Angst“ und „Scham“. Eine wichtige Frage ist, inwieweit diese „sozialisierenden Emotionen“ an der Herausbildung eines kultur-spezifischen Emotionsrepertoires beteiligt sind.

Schlüsselwörter

Sozialisation von Emotionen Emotionale Entwicklung Folk models Kulturvergleich Taiwan Madagaskar

\section{Learning (by) feeling: socialization and development of emotions in cross-cultural studies}

\begin{abstract}
Research on socialization and ontogeny of emotions has been widely neglected in social and cultural anthropology. In contrast, developmental psychologists are occupied intensively with this subject but the majority of their studies focus on Euro-American societies and thus do not explain intercultural differences. In this article we compare the socialization of emotions in two non-western societies: the Bara in Madagascar and the Tao on the Taiwanese Island of Lanyu. It will be illustrated how folk models of person, emotion, and development interrelate with local child rearing practices. In both societies sanctioning strategies are used by caregivers who operate with high levels of emotional arousal. While "fear" is induced among the
\end{abstract}


Bara, in the Tao's case the evoked emotions are "anxiety" and "shame”. An important question, among others, is to what extent these "socializing emotions" play a role in the development of a culture-specific emotional repertoire.

Keywords

Socialization of emotion Emotional development Folk models Cultural comparison Taiwan Madagascar

\section{Einleitung}

Die in der Biologie des Menschen angelegten basalen emotionalen Kapazitäten oder Affekte werden in unterschiedlichen Kulturen und Gesellschaften auf sehr differente Weise ausgeprägt, mit Bedeutungen und Wertungen belegt und in komplexe „Gefühlsregeln“ eingebettet, die vorschreiben, wer welche Emotionen wann und mit welcher Intensität empfinden und wem gegenüber wie aus- oder unterdrücken sollte. In der sozial- und kulturwissenschaftlichen Emotionsforschung wird die enorme inter- und intragesellschaftliche Vielgestaltigkeit emotionaler Verhaltens- und Ausdrucksweisen zwar mehrheitlich als Ergebnis komplexer bio-kultureller und psycho-sozialer Interaktionsprozesse gesehen, es mangelt jedoch an Studien, die diese Wechselprozesse systematisch und in fachübergreifender Form in den Blick nehmen. Besonders eklatant ist der Mangel an Studien, die sich mit der Sozialisation von Emotionen beschäftigen, d. h. sich der Frage widmen, wie Kinder in unterschiedlichen sozialen und kulturellen Kontexten das emotionale Repertoire ihrer jeweiligen Gemeinschaft erlernen. So lassen sich z. B. innerhalb der Ethnologie, die sich dezidiert der kulturellen Diversität von Emotionen widmet, keine Arbeiten finden, die sich mit der emotionalen Entwicklung und Erziehung von Kindern in verschiedenen Kulturen beschäftigen. ${ }^{1}$

In der Entwicklungspsychologie bildet dagegen der Zusammenhang zwischen Sozialisation und emotionaler Entwicklung ein etabliertes Thema, allerdings bezieht sich das Gros der entsprechenden empirischen Studien auf den euro-amerikanischen Kontext und hier primär auf Untersuchungen innerhalb der Mittelschichten (Holodynski 2004, 2009; Holodynski und Friedlmeier 2006; Shiman und Zehman 2001). ${ }^{2}$ Lediglich zu einzelnen Aspekten wie z. B. dem Einfluss von mütterlichen Erziehungsstilen auf die kindliche Regulationsfähigkeit von Emotionen liegt interkulturelles Datenmaterial vor (Friedlmeier und Trommsdorff 1999; Cole et al. 2002; Eisenberg et al. 2001). Vergleichsweise wenige Studien konzentrieren sich explizit auf den Zusammenhang zwischen Erziehungspraktiken und emotionaler Entwicklung, erwähnenswert sind hier v. a. die auf China (bzw. Taiwan) bezogenen Studien von Chang et al. (2003), Fung (1999) sowie Lieber et al. (2006). Doch auch diese Arbeiten aus dem Bereich der kulturvergleichenden Entwicklungspsychologie betrachten nur einzelne, aus dem Gesamtkontext gelöste Aspekte und nähern sich dem Zusammenhang von Kultur und Emotionsentwicklung nicht aus einer holistischen Perspektive an. Es mangelt somit auch in der kulturvergleichenden Entwicklungspsychologie an Studien, die gezielt die Interdependenzen zwischen kulturspezifischen Wertvorstellungen, Emotionskonzeptionen, Erziehungskonzepten und -praktiken sowie sozio-ökonomischen Strukturen und Bedingungen betrachten (Trommsdorff 2003).

Die skizzierten Forschungslücken innerhalb der ethnologischen sowie entwicklungspsychologischen Emotionsforschung gaben den Anstoß für die Konzeption eines bi-disziplinären Forschungsprojektes zur „Sozialisation und Ontogenese von Emotionen im Kulturvergleich“. .3 Das primäre Ziel dieses Projektes bestand zunächst darin, empirisches Material zu kulturspezifischen Formen der Emotionssozialisation zu erheben, um eine solide - und bisher fehlende - Datenbasis zur Beantwortung der fachübergreifenden Frage nach kulturbedingten Unterschieden in der Emotionsentwicklung zu schaffen. Im Rahmen parallel durchgeführter, jeweils über zwölfmonatiger ethnologischer Feldstudien in drei 
unterschiedlichen Kulturen wurde untersucht, auf welche Art und Weise Kinder das Emotionsrepertoire ihrer jeweiligen Gesellschaft erwerben und welche expliziten sowie impliziten kulturellen Transmissionspraktiken und Erziehungsmethoden hierbei eine Rolle spielen. Als Vergleichskulturen wurden die Minangkabau in West-Sumatra/Indonesien, die Bara im Süden von Madagaskar sowie die Tao auf der taiwanischen Insel Lanyu ausgewählt. Alle drei Gesellschaften weisen gravierende Unterschiede in ihrer Sozialstruktur, verwandtschaftlichen Organisation, Ökonomie, religiösen Orientierung sowie den Graden formaler (Schul-) Bildung auf, weshalb deutliche Differenzen in den jeweiligen Sozialisationsverläufen zu erwarten waren. Der „kleinste gemeinsame Nenner“, der diese Kulturen verbindet, ist ihre linguistische Zugehörigkeit zur west-austronesischen Sprachfamilie.

Aus diesem noch laufenden Forschungsprojekt soll im vorliegenden Beitrag ein Teilaspekt präsentiert werden, der den Zusammenhang zwischen emotionalisierenden, d. h. mit einem hohen emotionalen Erregungspotential arbeitenden Erziehungspraktiken wie Ängstigen, Beschämen, Loben, Verspotten, Aufziehen etc. und emotionaler Entwicklung fokussiert. Die Konsequenzen, die sich aus dem kulturspezifischen Einsatz emotional erregender Erziehungsmethoden für die Ausdifferenzierung von Emotionen innerhalb der Ontogenese ergeben, stellen eine emotionstheoretisch hochrelevante, aber bisher kaum untersuchte Thematik dar. Aus Platzgründen beschränken wir uns darauf, diese Fragestellung hier nur in Bezug auf zwei der von uns untersuchten Gesellschaften zu diskutieren: die Bara (Madagaskar) und die Tao (Taiwan).

Wir werden nachfolgend zunächst einige theoretische Grundüberlegungen sowie unser methodisches Vorgehen skizzieren, um dann ausgewählte Ergebnisse unserer Feldstudien in Madagaskar und Taiwan zu präsentieren.

\section{Theoretische Überlegungen}

Der Ausgangspunkt unserer Überlegungen stützt sich auf den Artikel Universals of Child Rearing der amerikanischen Kognitionsethnologin Naomi Quinn, der 2005 in Anthropological Theory erschienen ist. In dieser wegweisenden Studie hat Quinn neurowissenschaftliche Erkenntnisse über Lernprozesse zum Anlass für ein „close reading“ ethnografischer Texte genommen, welche Erziehungspraktiken in verschiedenen Kulturen beschreiben. Sie hat sich intensiv mit neurowissenschaftlichen Erkenntnissen über Lernprozesse - v. a. mit den Arbeiten des Hirnforschers LeDoux (2002) - auseinandergesetzt und diesen Studien vier zentrale Mechanismen entnommen, die Lernprozesse aus neurologischer Sicht außerordentlich effektiv machen, da sie zu besonders dauerhaften synaptischen Verbindungen führen. Es handelt sich hierbei um a) Prädispositionales Priming (predispositional priming), b) Konstanz der Erfahrung (experiential constancy), c) Billigung/Missbilligung (approval/disapproval) und d) emotionale Erregung (emotional arousal). Vor diesem Hintergrund hat Quinn sozialisationsbezogene ethnologische Studien wie z. B. die Arbeiten von Miller et al. (1996, 1997) zur taiwanesischen Mittelschicht, von Lutz (1988) zu den Ifaluk, von Briggs $(\underline{1982}, \underline{1998})$ zu den Inuit und von LeVine und LeVine (1966) zu den Gusii in Kenya etc. durchgesehen. In allen Texten hat sie klare Belege für die Anwendung dieser vier Erziehungstechniken gefunden, was diese zu starken Universalienkandidaten avancieren lässt und ihre - von der Neurowissenschaft behauptete - Effektivität zu bestätigen scheint. Quinns Studie deutet also an, dass in den unterschiedlichsten Kulturen mit den unterschiedlichsten sozialen Strukturen, Verhaltensstandards, Wertsetzungen und damit auch Erziehungszielen ein limitiertes (neuronal „sinnvolles“) Set von Erziehungspraktiken zum Einsatz kommt, um Kinder den jeweiligen gesellschaftlichen Standards entsprechend zu sozialisieren bzw. „for rearing children to be valuable adults“ (2005, S. 490). 
In unserem Beitrag knüpfen wir v. a. an Quinns Überlegungen zum Lernmechanismus der emotionalen Erregung im Rahmen von kulturspezifischen Erziehungspraktiken an. Sie hebt hervor, dass Erfahrungen, die im Zustand emotionaler Erregung gemacht werden, zu besonders dichten neuronalen Bahnen führen, was mit den hierdurch ausgelösten biochemischen Prozessen zusammenhängt. ${ }^{4}$ Affektiv erregende Erziehungspraktiken bewirken demnach, dass sich einem Kind die hiermit verbundenen Lektionen besonders fest und dauerhaft einprägen.

Wir gehen davon aus, dass emotionalisierende Erziehungspraktiken nicht nur eine zentrale Rolle bei der Werte- und Normsozialisierung spielen, sondern zugleich auch die emotionale Entwicklung entscheidend beeinflussen. Da Erziehungsstrategien wie Ängstigen, Verunsichern, Aufziehen, Beschämen oder Loben auf unterschiedlichen emotionalen Qualitäten basieren und je nach Kultur verschiedene Erziehungsstrategien dominieren können, dürfte die jeweils vorherrschende Erziehungspraxis zu einer kulturspezifischen Emotionsentwicklung beitragen.

Emotionen, die durch emotionalisierende Erziehungspraktiken direkt induziert werden und unmittelbar an der Sozialisation beteiligt sind, indem sie Individuen dazu veranlassen, sich an den Werten und Normen ihrer Kultur zu orientieren, nennen wir sozialisierende Emotionen. Neben der Induktion einer sozialisierenden Emotion kann es in vielen Fällen allerdings auch zu weiteren emotionalen Reaktionen auf Seiten des Kindes kommen, die nicht von Bezugspersonen intendiert sind. Strategien der Beschämung oder des Ängstigens können nicht nur Scham und Angst als sozialisierende Emotionen, sondern bspw. auch Traurigkeit oder Wut hervorrufen. Diese bezeichnen wir als sozialisierte Emotionen.

Folgende Fragen und Untersuchungsebenen werden von uns in diesem Beitrag erörtert: 1 .

Indigene Entwicklungstheorien: Welche Vorstellungen bestehen in den untersuchten Gesellschaften über die kindliche Entwicklung im Allgemeinen und die Emotionsentwicklung im Besonderen und welche Entwicklungsaufgaben resultieren daraus? Welche Emotionsdisplays sind bei Kindern in welchem Alter und in welchen Kontexten sozial erwünscht bzw. unerwünscht?

2.

Indigene Erziehungspraktiken: Welche Erziehungsstile und -methoden herrschen in der jeweiligen Kultur vor und mit welchen emotionalen Qualitäten arbeiten diese? Auf welche Weise führen diese Erziehungsmethoden zur Herausbildung von sozialisierenden Emotionen einerseits und sozialisierten Emotionen andererseits?

\section{Methodisches Vorgehen}

Um den jeweiligen kulturellen Besonderheiten gerecht zu werden, verfolgten wir einen relativ offenen und explorativen methodischen Ansatz. Mit dem Ziel, die Vergleichbarkeit der Ergebnisse zu gewährleisten, haben wir jedoch im Vorfeld eine Reihe von Methoden ausgewählt, von denen die folgenden letztlich in beiden Kulturen angewendet werden konnten:

1.

Teilnehmende Beobachtung zur Erschließung allgemeiner sozialer und kultureller Zusammenhänge.

2.

Haushalts-Surveys zur Erhebung soziodemographischer Daten, die Rückschlüsse auf die sozialen und häuslichen Lebensbedingungen der Kinder ermöglichen.

3. 
Erhebung der indigenen Emotionsbegriffe mittels freelisting Verfahren und Erhebung von Kurzgeschichten zu sämtlichen genannten Emotionswörtern zur Bedeutungsanalyse des lokalen Emotionsvokabulars (natural definitions-Ansatz).

4.

Unstrukturierte, semistrukturierte und strukturierte Interviews mit Erwachsenen zur Erhebung lokaler Vorstellungen von Emotion, kindlicher Entwicklung und Erziehung sowie mit Kindern zu ihren Erziehungserinnerungen.

5.

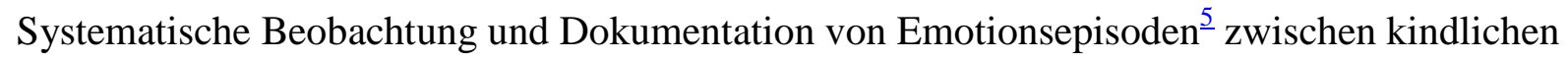
Akteuren sowie zwischen Kindern unterschiedlichen Alters und ihren Bezugspersonen.

Das so erhobene Datenmaterial wurde (bzw. wird) in gemeinsamer Abstimmung v. a. nach den Prinzipien der Grounded Theory (Strauss und Corbin 1990) ausgewertet. Das Material zu den Tao konnte aufgrund des erst im August 2011 beendeten Feldaufenthaltes noch nicht vollständig analysiert werden, so dass die hier aufgeführten Ergebnisse als vorläufig angesehen werden müssen.

\section{Sozialisation von Emotionen bei den Bara (Madagaskar) ${ }^{6}$}

Die etwa eine halbe Million Menschen umfassende ethnische Gruppe der Bara siedelt vorwiegend im südlichen Hochland von Madagaskar in einer trockenen Gras- und Baumsavanne. Reisanbau und Viehhaltung bilden die Grundpfeiler der wirtschaftlichen Aktivitäten - Reis dient als Hauptnahrungsmittel, Rinder werden als „Wertspeicher“ genutzt und sind zudem von großer sozialer und religiöser Bedeutung. Im Unterschied zu vielen anderen madagassischen Gruppen ist die Gesellschaft der Bara segmentär strukturiert, wobei relativ klar abgegrenzte Patrilineages die zentralen sozialen Einheiten darstellen. Idealerweise entspricht die Patrilineage der Bevölkerung eines Dorfes, doch in verhältnismäßig fruchtbaren Regionen teilen sich mehrere Verwandtschaftsgruppen eine Siedlung. Patrilineages umfassen i. d. R. drei bis vier Generationen lebender Menschen und - berücksichtigt man die emische Perspektive - zwei bis drei Generationen von Ahnengeistern, welche die zentralen religiösen Instanzen darstellen. Die sozialen Interaktionen innerhalb der Patrilineage orientieren sich am Senioritätsprinzip und der Verpflichtung zur Kooperation. Intersegmentäre Beziehungen folgen hingegen stärker dem Egalitätsprinzip und lassen offen ausgetragene Konflikte und Rivalität zu.

Nationalstaatliche Institutionen und moderne Infrastruktur sind in der Forschungsregion bislang wenig verbreitet. Im Zeitraum der Forschung existierten weder eine Straßenanbindung noch ein Schulsystem und auch christliche Kirchen waren nicht vorhanden. Seit einigen Jahren sind in der Nähe des Forschungsortes Gendarmen stationiert, die aber nicht als Organ des Staates, sondern vielmehr als individuelle Marodeure wahrgenommen werden. Industriell gefertigte Güter wie Kleidung, alkoholische Getränke, Zucker oder synthetische Medikamente sind in bescheidenem Ausmaß in der Region verfügbar.

\subsection{Kulturelle Modelle der Ontogenese und Erziehung}

Die Ontogenese wird in der Bara-Kultur als ein relativ kontinuierlicher Wandlungsprozess konzipiert, den Huntington als eine Reise vom mütterlichen Leib (als Fötus) in das väterliche Grab (als Ahnengeist) darstellt (1988, S. 28). Institutionell oder rituell markierte Statusübergänge wie etwa die Initiation in eine Altersgruppe fehlen vollständig und auch das kulturelle Bedeutungssystem sieht kaum Einteilungen in verschiedene Entwicklungsphasen vor. Lediglich für Säuglinge in den ersten drei Lebensmonaten existiert eine besondere Bezeichnung (zaza mena - rotes Kind), Kinder ab drei Monaten bis zur Geschlechtsreife werden durchgängig als zaza bezeichnet. Orientiert man sich zusätzlich an markanten 
Veränderungen der Interaktionsmuster, so lassen sich weitere Phasenübergänge im Alter von knapp zwei Jahren (Entwöhnung) und im Alter zwischen vier und fünf Jahren (Beginn der dezidierten Normvermittlung) festmachen.

Die expliziten elterlichen Erziehungsziele kommen v. a. in dem kulturellen Konzept mahitsy zum Ausdruck. Mahitsy bezeichnet ein ideales und folglich in der Erziehung angestrebtes kindliches Verhalten, dass sich durch Fügsamkeit und die Bereitschaft zur Unterordnung auszeichnet. Konkret umfasst es folgende Facetten: Unwidersprochene Folgsamkeit gegenüber älteren Lineage-Mitgliedern, gestischer und verbaler Ausdruck der untergeordneten Position gemäß der Altershierarchie und eine emotionale Bereitschaft, ältere Mitglieder aus der eigenen Verwandtschaftsgruppe zu fürchten (matahotsy). Obwohl mahitsy i. d. R. zur Charakterisierung von Kindern verwendet wird, behalten die damit bezeichneten Verhaltens- und Einstellungsideale auch im Erwachsenenalter Gültigkeit. Selbst Angehörige der ältesten Generation sind davon nicht ausgeschlossen, da sie gegenüber den Ahnengeistern die Rolle von Junioren einnehmen.

\subsection{Entwicklungsphasen, Interaktionsmuster und Sanktionspraktiken}

Säuglingsphase (0-2): Die ersten beiden Lebensjahre sind durch eine körperlich enge, aber nicht exklusive Mutter-Kind-Beziehung charakterisiert. Diese basiert v. a. auf einem hohen Maß an Körperkontakt sowie rhythmischer Körperstimulation und einer großzügigen Befriedigung körperlicher Bedürfnisse. So werden Säuglinge nach Bedarf und häufig auch proaktiv, d. h. noch vor der Bedarfsäußerung, gestillt. Negative Emotionen treten v. a. aufgrund der nahezu permanenten körperlichen Verfügbarkeit der Mutter relativ selten auf und werden zudem umgehend und effektiv herunterreguliert. Schnelles Wachstum und motorische Entwicklung sowie Gesundheit stehen im Fokus der mütterlichen Bemühungen. Das Teilen positiver Emotionen und der explizite Ausdruck gegenseitiger Liebe spielt hingegen eine untergeordnete Rolle. Insgesamt dominiert ein proximaler gegenüber einem distalen Interaktionsstil (Keller 2007, S. 143).

Kleinkindphase (2-4/5): Das Abstillen im Alter von durchschnittlich knapp zwei Jahren zieht häufig eine relativ abrupte Distanzierung zwischen Müttern und Kleinkindern nach sich und leitet zugleich eine schnelle Eingliederung der Kleinkinder in die Gruppe der Gleichaltrigen ein. In der Regel geht dieser Ablösungsprozess mit der Geburt eines weiteren Kindes einher, dem die Mutter dann ihre volle Zuwendung schenkt. In dieser Entwicklungsphase machen Kinder größtenteils die ersten Sanktionserfahrungen, die häufig auch die körperliche Distanzierung von der Mutter forcieren. So können Kleinkinder einen Klaps erhalten oder gekniffen werden, wenn sie Mütter bei ihrer Arbeit stören, indem sie ihre körperliche Nähe suchen, Hausrat durcheinanderbringen oder Nahrungsmittel verschütten bzw. verunreinigen. Häufig werden sie parallel zu diesen Körpersanktionen dazu angehalten, mit ihren Altersgenossen zu spielen.

Kindheitsphase (4/5-14): Ab dem Alter von vier bis fünf Jahren wird von Kindern normkonformes Verhalten und Handeln sowie das regelmäßige Ausführen von Aufgaben im Rahmen der Nahrungszubereitung erwartet. Missachtung dieser Verhaltensanforderungen wird nun in systematischer Weise sanktioniert. Diese Interaktionsumstellung begründen Eltern häufig damit, dass Kinder ab diesem Alter die Fähigkeit erlangten, Normen zu verstehen und die Sanktion mit dem eigenen Normverstoß in Verbindung zu bringen. Während der gesamten Kindheit ist die Körpersanktion des Schlagens (mifofoky) das zentrale Erziehungsmittel. Schlagen wurde von sämtlichen Gesprächspartnern als vorzuziehende und wirksamste Sanktionsform dargestellt, am häufigsten als Reaktion auf kindliches Fehlverhalten genannt und als adäquate Sanktion für alle möglichen Formen von Normverstößen dargestellt. Aufgrund der Prävalenz des Schlagens gegenüber anderen 
Sanktionsformen steht die Praxis des Schlagens im Mittelpunkt der nachfolgenden

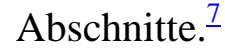

Jugendphase (ab 14): Ab dem Jugendalter wird die Sanktionierung durch Schlagen eingestellt, weil die Gefahr gesehen wird, dass sich die körperlich ebenbürtigen Jugendlichen zur Wehr setzen. Mädchen dürfen ab der Geschlechtsreife nicht mehr von verwandten Männern geschlagen werden, weil dies eine Verletzung der zwischengeschlechtlichen Meidungsgebote bedeuten würde. In der Vorstellung der Akteure wird die Sanktionsverantwortung in diesem Alter von Ahnengeistern übernommen, deren Zorn Krankheiten oder andere Formen des Unglücks auslösen. Die Betroffenen befürchten einen schleichenden und letztlich tödlichen Verlauf der so verursachten Krankheiten, sehen aber die Möglichkeit, die Ahnen durch Darbietungen von Alkohol- oder Rinderopfern zu besänftigen und damit die Krankheitsursache aufzuheben sowie die Lebensbedrohung abzuwenden.

\subsection{Die Sanktionspraxis des Schlagens}

Im Unterschied zur Körperstrafe des Klapsens beinhaltet die Sanktionsform des Schlagens i. d. R. eine Reihe von einzelnen Schlägen, die meist mit einem Hirtenstock ausgeführt werden. Zumeist unterstreicht die sanktionierende Person die Schläge durch einen intensiven Wutausdruck (meloky) und droht dem Kind damit, es im Fall von wiederholtem Fehlverhalten heftiger zu schlagen oder gar zu töten. Veranlasst wird diese Form der Körperstrafe nach Auskunft von Eltern und von Kindern am häufigsten durch a) die Weigerung, aufgetragene Aufgaben auszuführen, b) Widerworte oder Zurückschlagen als Reaktion auf eine Sanktion, c) respektloses Verhalten gegenüber älteren Verwandten, d) aggressives Verhalten gegenüber jüngeren Geschwistern und e) durch Stehlen von Hühnern oder Nahrungsmitteln. Dabei ist zu betonen, dass diese Verhaltensweisen nur dann konsequent als Normverstöße sanktioniert werden, wenn sie sich auf die patrilineare Verwandtschaftsgruppe des Kindes beziehen. Mädchen und Jungen können gleichermaßen der Körperstrafe unterzogen werden, allerdings ist die Vorstellung verbreitet, dass Mädchen weniger stark und seltener geschlagen werden müssen, da sie sich leichter fürchten würden. Am häufigsten führen Väter die Körpersanktion des Schlagens aus, aber auch andere männliche Mitglieder der Patrilineage können vertretungsweise die Rolle des Sanktionierenden übernehmen. Besonders bei kleineren Kindern treten auch Mütter in dieser Rolle auf.

In der Regel werden Kinder unmittelbar nach der Körperstrafe von ihren Müttern oder anderen weiblichen Verwandten getröstet (mitambitamby), indem diese bspw. den Kopf des Kindes streicheln, es vor der sanktionierenden Person in Schutz nehmen oder ihm deutlich machen, dass sich die Sanktion lediglich auf sein Fehlverhalten bezieht und es weiterhin geborgen ist. Die Rollen des Sanktionierenden und Tröstenden werden keinesfalls von derselben Person übernommen und sind für jedes Kind auf bestimmte Personen festgelegt.

\subsection{Körpersanktion und Emotionsentwicklung}

Aus den Befragungen von Erwachsenen zu ihrer Sanktionspraxis sowie von Kindern und Jugendlichen zu ihren Sanktionserfahrungen geht eindeutig hervor, dass die Sanktion des Schlagens intensive Furcht (matahotsy) auslöst. Es ist davon auszugehen, dass die wiederholte Erfahrung geschlagen zu werden und die damit einhergehende akute Furcht vor Schmerzen und körperlicher Beeinträchtigung zur Herausbildung einer kulturspezifischen Furchtdisposition beiträgt. Aufgrund dieser Furchtdisposition antizipiert das Kind den Zorn einer Autoritätsperson, die Bestrafung durch Prügel sowie die körperlichen Schmerzen als Folge eines Normverstoßes, für welchen es in der Vergangenheit sanktioniert wurde. Diese Antizipation löst Furcht aus und motiviert so das Kind, die entsprechende Norm auch im 
Widerspruch zu seinen etwaigen anderen Motiven und Emotionen zu befolgen, um der Sanktion zu entgehen.

Im Übergang zum Jugendalter durchläuft die Furchtdisposition offenbar eine entscheidende Transformation: Furcht wird in diesem Alter zunehmend aufgrund der Antizipation von Sanktionen durch Ahnengeister ausgelöst und auf diese Weise mit religiösen Vorstellungen verknüpft. Das heißt, nicht mehr aufgrund der Sanktionierung durch reale Personen, sondern allein durch die Imagination bestrafender Ahnengeister wird nun Furcht ausgelöst. Diese Umwandlung ist für die Funktionalität der Furchtdisposition im Erwachsenenalter entscheidend, da aufgrund der physischen Ebenbürtigkeit ab der Jugend reale Autoritätspersonen die Körpersanktion einstellen und folglich nicht mehr als solche, sondern lediglich als Repräsentanten der Ahnen gefürchtet werden.

Die Körpersanktionen tragen also zur Herausbildung der Emotionsdisposition matahotsy bei, die als eine kulturspezifische Form der Furcht das Individuum veranlasst, die sozialen Normen der Bara-Gesellschaft eigenständig zu befolgen. Somit ist matahotsy als eine zentrale sozialisierende Emotion in der Bara-Gesellschaft anzusehen.

Bemerkenswert ist, dass in den Interviews zu Sanktionserfahrungen kaum Gefühle der Scham oder der Erniedrigung als Folge der Körpersanktion berichtet wurden. Dies dürfte u. a. mit folgenden Vorkehrungen zusammenhängen: a) Die Körpersanktion wird unter Ausschluss Unbeteiligter, insbesondere Gleichaltriger durchgeführt. b) Gemäß dem Senioritätsprinzip besteht von vornherein ein Unterordnungsverhältnis zwischen der sanktionierenden und der betroffenen Person. c) Die Betroffenen werden von einer nahestehenden Person auf die beschriebene Art und Weise getröstet. Potentielle Nebeneffekte der Körperstrafe wie die Erfahrung von Erniedrigung oder sozialem Ausschluss können auf diese Weise offenbar minimiert werden.

Kinder und Jugendliche berichten jedoch durchgehend, dass sie während einer Züchtigung neben Furcht stets auch intensive Wut (maseky) gegenüber der Sanktionsperson empfunden hätten. Die wiederholte Erfahrung der Körpersanktion dürfte damit eine relativ hohe Wutoder Aggressionsbereitschaft fördern, was einem Review von Gershoff (2002, S. 541) zufolge eine generelle, kulturübergreifende Konsequenz der Körperstrafe zu sein scheint. Wut und Aggression sind allerdings nicht nur eine Reaktion auf die Körperstrafe, sondern oftmals auch der Anlass für ihre Anwendung. Wie die angeführten Anlässe der Körpersanktion zeigen, wird aggressives Verhalten in Abhängigkeit vom sozialen Kontext, in dem es auftritt, sanktioniert. Diese auf Wut und Aggressionen bezogene Sanktionspraxis dürfte zu einer Ausdifferenzierung der zunächst unspezifischen Wut in drei Subgruppen beitragen, was abschließend nur kurz skizziert werden kann:

1.

Aggressives Verhalten gegenüber dem sanktionierenden Erwachsenen gilt ab dem Alter von ca. fünf Jahren als inakzeptabel und wird durch eine Fortsetzung oder Wiederholung des Schlagens konsequent sanktioniert. Auf diese Weise lernt das Kind, seine aggressiven Tendenzen gegenüber Autoritätspersonen zu unterdrücken und durch ein weniger offensives Ausdrucksverhalten zu ersetzen. Dies dürfte zur Ausbildung einer Gruppe von ÄrgerEmotionen beitragen, die sich dadurch auszeichnen, dass sie auf Autoritätspersonen gerichtet sind und in ein spezifisches Ausdrucksverhalten anstelle einer aggressiven Handlung münden. Entsprechungen hierfür auf der Konzeptebene sind bspw. die Emotionsbegriffe mimotso und mihindrotsy. Während mimotso mit einem Spitzen der Lippen assoziiert ist und damit dem deutschsprachigen „Schmollen“ ähnelt, geht mihindrotsy mit einem Zusammenziehen und Anheben der Augenbrauen einher, wofür offenbar kein Emotionswort in der deutschen Sprache existiert.

2.

Aggressives Verhalten gegenüber nichtverwandten Gleichaltrigen wird i. d. R. nicht sanktioniert und teilweise werden Kinder sogar von Bezugspersonen dazu ermutigt, sich 
körperlich gegen Gleichaltrige durchzusetzen. Zudem berichten einige Kinder, ihre Wut infolge der Körpersanktion an anderen Kindern ausgelassen zu haben. Beides dürfte zur Ausbildung einer Reihe von aggressiven Wut-Emotionen beitragen, von welchen eine als may fo bezeichnet wird, was wörtlich heißes Herz bedeutet.

3.

Wut und daraus resultierende Handlungen gegenüber jüngeren Geschwistern werden sanktioniert, wenn sie einen aggressiven Charakter haben und unberechtigt sind, nicht aber, sofern sie sich in einem angemessenen Maß auf Fehlverhalten der Jüngeren beziehen. Zusammen mit dem Lernvorgang der Rollenübernahme dürfte dies die Herausbildung von sanktionsmotivierenden Ärger-Emotionen begünstigen, die auf der konzeptuellen Ebene u. a. durch den Begriff meloky fassbar werden, der sich mit Zorn im Sinne von gerechtfertigter Wut übersetzen ließe.

Die Praxis der körperlichen Züchtigung in der Bara-Gesellschaft begünstigt also nicht nur die Entwicklung einer spezifischen Furcht (matahotsy) als (selbst-) sozialisierende Emotion, sondern trägt auch zur Ausdifferenzierung von unspezifischer Wut in drei Cluster mit diskreten Wut- bzw. Ärgeremotionen als sozialisierte Emotionen bei. Die Emotionen des dritten Clusters motivieren sanktionierendes Verhalten wie die Körperstrafe und haben somit eine (fremd-) sozialisierende Funktion. Die emotionalen Konsequenzen der Körpersanktion begünstigen letztlich also auch eine Weitergabe der Praxis der Körperstrafe von einer Generation an die nächste.

\section{Sozialisation von Emotionen bei den Tao (Taiwan) ${ }^{\underline{8}}$}

Die Tao stellen mit insgesamt etwa 3500 Personen eine verhältnismäßig kleine Untersuchungsgruppe dar. Ihre Vorfahren besiedelten vor 800 Jahren von den nordphilippinischen Batan-Inseln aus die Vulkaninsel Lanyu, die sich etwa 75 km südöstlich von Taiwan befindet. Das Innere der Insel besteht aus steil aufragenden Bergen, die von subtropischem Regenwald überzogen sind. Im Sommer können Taifune eine vernichtende Wirkung entfalten. Die Tao pflanzen Taro und Süßkartoffeln in ihren Gärten an und betreiben Fischfang, dem große rituelle Bedeutung beigemessen wird. Im alltäglichen Leben spielt die Kernfamilie eine wichtige Rolle, sie bildet die zentrale Sozialeinheit. Diesbezüglich unterscheiden sich die Tao von den Bara, bei denen die Großfamilie dominiert. Die soziale Organisation weist egalitäre Züge auf, soziale Ränge oder politische Ämter sind unbekannt. Die Tao verfügen über schwach ausgeprägte Patrilineages, deren Mitglieder idealiter bei der Produktion von Nahrung kooperieren. Zwischen den verschiedenen Abstammungsgruppen bestehen rivalisierende Beziehungen, die in der Vergangenheit zu Gruppenkämpfen geführt haben. Aufgrund des pazifizierenden Einflusses der Polizei ist es jedoch in den letzten 30 Jahren zu keinen größeren Kampfhandlungen mehr gekommen. Heute sind die meisten Tao zum Christentum konvertiert. Der traditionelle Glaube an übelwollende Geister (anito), von denen eine ständige Gefahr ausgeht, ist jedoch nach wie vor sehr bedeutsam.

Während der japanischen Kolonialzeit (1897-1945) wurde eine Politik der Nichteinmischung betrieben, so dass die Tao ihren traditionellen Lebensstil bis in die zweite Hälfte des 20 .

Jahrhunderts weitgehend beibehalten konnten. Ein rasanter gesellschaftlicher Umbruch setzte erst um 1970 ein, als sich immer mehr junge Tao in die Arbeitsmigration nach Taiwan begaben, wo sie als ungelernte Arbeitskräfte in der Landwirtschaft und im Baugewerbe Geld verdienen konnten. Bei ihrer Heimkehr führten sie zuhause die monetäre Wirtschaftsform und den bis dahin unbekannten Alkohol ein. Ein modernes Schulsystem besteht erst seit den 1960er Jahren. 1982 wurde unter dem Vorwand, eine Fischkonservenfabrik zu errichten, eine Atommülllagerstätte auf Lanyu gebaut, in der Taiwan trotz massiver indigener Proteste bis heute seine radioaktiven Abfälle deponiert. Die Kompensationszahlungen des nationalen 
Energiekonzerns Taipower, der auch als ein wichtiger Arbeitgeber fungiert, sind heute eine bedeutende Einnahmequelle der sechs Inselgemeinden.

\subsection{Kulturelle Modelle der Ontogenese und Erziehung}

Kindheit (no kakanakan) wird von den Tao als ein kontinuierlicher Entwicklungsprozess verstanden, der nicht in abgrenzbare Phasen unterteilt werden kann. Die Tao gehen davon aus, dass die Seele eines Kindes zunächst „schwach“ und hochgradig anfällig für diesseitige sowie jenseitige Gefahren ist und sich erst nach und nach im Körper verfestigt bzw. erhärtet, wodurch sich ihre Anfälligkeit reduziert. Die noch weichen Seelen von Neugeborenen und kleinen Kindern können z. B. durch die Wahrnehmung eines Geistwesens (anito) derartig erschreckt werden, dass sie aus dem Körper des Kindes austreten und davonfliegen (somalap so pahad). Ein Kind, das seine Seele verloren hat, weint ununterbrochen und ist nicht mehr zu beruhigen. Die ersten Lebensmonate verbringen Kinder deshalb im Schutz des Hauses, wo sich eine Bezugsperson - zumeist die Mutter - immer in ihrer Nähe aufhält. $\stackrel{9}{ }$ Säuglinge schlafen in einer aus alten Reissäcken gefertigten Wiege und dürfen unter keinen Umständen allein gelassen werden. Sobald das Kind mit 7-8 Monaten selbstständig sitzen kann, wird ein Ritual durchgeführt, bei dem es ein Schutzamulett erhält, welches es weniger anfällig für Geister machen soll. Von nun an wird es gelegentlich mit nach draußen genommen, bleibt aber nach wie vor immer in der Nähe der Mutter.

In den ersten beiden Lebensjahren besteht den Tao-Konzeptionen gemäß die Hauptaufgabe der Eltern darin, ihre Kinder zu nähren und vor den vielfältigen Gefahren, die auf sie lauern, zu beschützen. Je älter und motorisch sicherer sie werden und je mehr ihre Seele sich verfestigt, desto größer werden die Bewegungsspielräume der Kinder. Sie verlassen nun zunehmend die Obhut der Erwachsenen und erwerben die unterschiedlichsten Fertigkeiten durch Beobachtung und selbstständiges Ausprobieren. Erwachsene mischen sich nur selten in kindliche Explorationen ein, vor allen Dingen aber steuern sie diese nicht gezielt. Erst wenn Kinder anfangen, die „Dinge zu verstehen“, setzt eine explizite Unterweisung in kulturspezifische Wissens- und Arbeitsformen ein. Heute geht man davon aus, dass Heranwachsende hierzu erst mit 12 Jahren in der Lage sind, wenn sie die 6-stufige Grundschule abschließen. In früheren Zeiten, als die Lebensweise der Tao noch primär durch die Subsistenzwirtschaft geprägt war, beteiligten sich Kinder bereits mit etwa 8 Jahren regelmäßig an der Nahrungsmittelproduktion. Die aktive Mithilfe galt als Indikator für die „geistige Reife“ der Kinder und damit als geeigneter Zeitpunkt, um mit systematischer Unterweisung und Belehrung der Heranwachsenden zu beginnen. Aufgrund der Ganztagsschule ist Kindern heute das Mithelfen auf den Feldern kaum noch möglich, wodurch sie viele überlieferte Fertigkeiten sowie Wissensformen nicht mehr erwerben können.

\subsection{Entwicklungsphasen, Interaktionsmuster und Sanktionspraktiken}

Auch wenn die Ontogenese aus emischer Sicht nicht in klare, explizit benannte Phasen unterteilt wird, so lassen sich aus etischer Perspektive dennoch drei Entwicklungsphasen unterscheiden. Diese Phasen lassen sich an veränderten Interaktionsformen der Erwachsenen mit den Kindern festmachen, die sich an der kognitiven Reifung des Kindes zu orientieren scheinen, d. h. an der Entwicklung seines Sprachverständnisses und der Herausbildung einer theory of mind, die es dem Kind ermöglicht, sich in andere Personen oder Wesen hineinzuversetzen.

Säuglingsphase (0-2): Die ersten beiden Lebensjahre des Kindes zeichnen sich durch eine enge Mutter-Kind-Beziehung aus. Die Mutter umsorgt das Kind auf proaktive Weise, indem sie auf alle seine physischen Bedürfnisse achtgibt und sie zu erfüllen versucht, bevor sich das 
Kind durch Weinen bemerkbar machen muss. Von Kindern wird allgemein erwartet, dass sie sich ruhig verhalten (mahanang), den Autoritätspersonen gehorchen (mamizing) und sie achten und sich ihnen gegenüber respektvoll verhalten (kanig). Bereits mit knapp einem Jahr werden quengelige Kinder durch wütende Gesichter und drohendes Klatschen auf den Holzboden diszipliniert. Im Verlauf des zweiten Lebensjahres bekommen Kinder bei Normüberschreitungen Klapse auf die Hand oder gegen das Bein. In dieser Zeit wird auch die drohend erhobene rechte Hand eingeführt, die Bereitschaft zu schlagen ausdrücken soll. Weibliche Bezugspersonen imitieren mit ihren Fingern „Krabbelspinnen“, die sich bedrohlich auf Kleinkinder zuwütenden Grimmassen. Kinderbewegen. Männliche Bezugspersonen halten kleine Kinder dicht vor ihr Gesicht und verziehen dieses abwechselnd zu lächelnden und wütenden Grimassen. Kinder, die gerade laufen gelernt haben, werden manchmal von ihren Bezugspersonen mit dem Finger in den Rücken oder ins Bein gepiekt, doch wenn sie sich dann irritiert umdrehen, ist hinter ihnen niemand zu sehen. Dieser Vorgang wird zur Belustigung der Versammelten mehrmals wiederholt, bis sich das Kind ganz still verhält. Bezugspersonen verhalten sich gegenüber kleinen Kindern oft auf eine irritierende, ambivalente und Angst einflößende Weise.

Kleinkindphase (2-4): Wenn Kinder zwei bis drei Jahre alt werden, entfernen sie sich von ihren Müttern. Dies ist ein allmählicher Prozess, der mit einer sukzessiven Erweiterung ihres Aktionsradius einhergeht. Trotzdem werden sie von Müttern oder anderen Bezugspersonen in diesem Alter immer im Blick behalten. Ab drei Jahren können Kinder von ihren älteren Geschwistern zu kurzen Ausflügen oder Erledigungen innerhalb des Dorfes mitgenommen werden.

Das Belehren (nanaon) gilt bei den Tao als ideale Erziehungsmethode. Worte dürfen nie einfach nur dahergesagt werden, da sie in Form von Segnungen und Flüchen auf das Geschehen Einfluss nehmen können. Sobald Kinder mit etwa zwei Jahren ein erweitertes Sprachverständnis erwerben, werden sie darüber belehrt, was sie alles nicht tun dürfen. Mit zunehmendem Alter werden die Ermahnungen immer komplexer und nehmen schließlich aus der Perspektive einer westlichen Mittelschicht - den Charakter von Drohungen an. So sagt man zu kleinen Kindern z. B.: „Geh nicht zu nah an den Käfig heran oder das Huhn wird dir die Augen auspicken!“; „Leg das Messer aus der Hand oder du wirst dich verletzen!“ Diesen Sprüchen ist gemein, dass sie ein Szenario entwerfen, in dem die körperliche Unversehrtheit der Kinder bedroht ist. Tritt irgendwann eine der Prophezeiungen tatsächlich ein, wird dies dann sowohl von den Bezugspersonen als auch den Kindern selber als Strafe für die Missachtung der Belehrungen gewertet. Bedeutsam ist, dass hier nicht die Bezugspersonen, sondern die gesamte natürliche und supernatürliche Umwelt des Kindes als potentielle Sanktionierungsinstanz auftritt.

Kleine Kinder sollen sich immer in der Nähe des Dorfes aufhalten, was sicherlich eine berechtigte Forderung ist, wenn man sich die gefährliche naturräumliche Umgebung Lanyus vergegenwärtigt: Die Küste besteht aus messerscharfem Korallengestein und in der Wildnis der Bergwelt kann man sich leicht verlaufen und den Weg in die menschliche Ansiedlung nicht mehr zurückfinden. Die Erwachsenen warnen ihre Kinder deshalb häufig vor übelwollenden Geistern, die dort lauern und nach ihren Seelen trachten. Für die Tao, die von der Existenz der übernatürlichen Geschöpfe überzeugt sind, stellen diese Warnungen eine Form des Belehrens dar, als „Ängstigen“ werden sie nicht gewertet, wie in den Interviews zu den Erziehungsmethoden deutlich wurde.

Von zwei Jahren an, also mit beginnendem Sprachverständnis, wird mit Kleinkindern geschimpft. Eine verbreitete Form des Schimpfens ist das Anblaffen (ioya). Dabei positioniert sich der Sanktionierende vor dem Kind, beugt seinen Oberkörper mit erhobener rechter Hand nach vorne und lässt im Stakkato eine Schimpftirade auf das Kind herab. Die Stimme bleibt nie lange erhoben und auch die Dauer des Ausschimpfens ist eher kurz. Die sanktionierende Person hat ihren Ärger (somozi) dabei unter Kontrolle. Anblaffen (ioya) muss von einem 
länger anhaltendem Anschreien (amlololos) unterschieden werden. Letzteres deutet auf einen Kontrollverlust der Bezugsperson hin und sollte vermieden werden, obwohl es in der Realität durchaus vorkommt. Eine typische kindliche Reaktion auf das Anblaffen ist das Weglaufen, das vom fünften Lebensjahr an vermehrt zu beobachten ist. In der Regel nehmen Kinder sofort Reißaus, sobald einer der Erwachsenen seine rechte Hand zu einer Drohgebärde erhebt. Anlässe für das Anblaffen liegen vor, wenn Kinder zum wiederholten Mal den

Aufforderungen der Erwachsenen nicht Folge leisten oder Haushaltsgegenstände beschädigen. Man kann oft beobachten, wie ein älteres Kind oder eine erwachsene Person sich von hinten an ein Kleinkind heranschleicht und es dann plötzlich mit beiden Händen am Rücken berührt. Wenn das Kind eine schreckhafte Reaktion zeigt, wird es von den umstehenden Personen ausgelacht. Auch Zweijährige, die sich wegen eines lauten Geräusches erschrecken und zu weinen anfangen, werden von den Anwesenden verspottet. Bereits mit zwei bis drei Jahren haben sich die meisten Kinder in solchen Situationen unter Kontrolle und lassen sich kaum eine Reaktion anmerken. Die Tao glauben, dass sich die Intensität der empfundenen Angst (maniahey) im Laufe des Lebens immer weiter verringert. Diese Vorstellung korreliert mit der Konzeption von Kindheit als einem Aushärtungsprozess der Seele.

Kindheitsphase (ab 4): Mit vier Jahren verbringen Kinder die meiste Zeit mit ihren Altersgenossen. Die Interaktion mit den Eltern reduziert sich auf das Einnehmen der Mahlzeiten. Am Nachmittag nach der (Vor-) Schule bleiben ihnen nur wenige Stunden zum Kräftemessen und zum Ausüben von Geschicklichkeitsübungen. Früher mussten sie vor Einbruch der Dunkelheit wieder zuhause sein, da nach indigener Vorstellung dann die anitoGeister vermehrt in der Umgebung des Dorfes erscheinen. Seitdem die Dörfer Lanyus jedoch über eine Elektrizitätsversorgung verfügen, sind die Gassen hell erleuchtet, so dass die Kinder auch noch abends draußen spielen können.

Es kommt häufig vor, dass eines der Kinder von seinen Spielkameraden „schikaniert“ wird (jyasnekan ${ }^{10}$ ). Die Bandbreite dieses Verhaltens reicht über Verspotten, Einsperren, gewaltsame Entwendung von Kleidung, Steine werfen, Verprügeln bis hin zum gewaltsamen Untertauchen beim Spielen in der Meeresbucht. Ältere Kinder drohen den jüngeren Schläge an, wenn diese ihnen nicht bestimmte Snacks aus dem Dorfladen kaufen, oder sie nehmen ihnen ihr Kleingeld ab. Ein Kind kann sich nie sicher sein, ob es nicht im nächsten Moment von seinen Altersgenossen geärgert wird. Jede Unachtsamkeit kann zum Verhängnis werden. Oft bekommen die Erwachsenen gar nicht mit, was sich in einer Kindergruppe abspielt. Sie greifen nur ein, wenn ernstliche Verletzungsgefahr für ein Kind besteht. Kinder machen in diesen Kontexten häufig die Erfahrung, dass sich plötzlich ihr gesamtes Umfeld gegen sie wendet, niemand ihnen zur Hilfe kommt und sie auf sich allein gestellt sind. Bereits Vierjährige haben ihre gesamte Umgebung ständig im Blick, um aufkommende Bedrohungen frühzeitig zu erkennen und meiden Alleinsituationen, die generell als gefährlich und unsicher empfunden werden.

Wenn ein schikaniertes Kind wütend (somozi) wird oder anfängt zu weinen (amlavi), wird es von seinen Peinigern ausgelacht. Generell gilt, dass negative Emotionen, zu denen auch Traurigkeit zählt, nicht gezeigt werden dürfen. Wenn vier- bis sechsjährige Kinder traurig (marahet so onowned) sind und ihre Tränen nicht mehr zurückhalten können, verkriechen sie sich hinter den Schweineställen oder einem am Straßenrand geparkten Auto, um im Verborgenen zu weinen.

Heute wird in vielen - jedoch nicht in allen - Familien die Erziehungsmethode der körperlichen Züchtigung (kabakbak) angewandt. Die Interviews zu den Erziehungserinnerungen älterer Personen belegen allerdings, dass Schlagen früher verpönt war. Man befürchtete, dass sich die Kindesseele erschrecken und davonfliegen könnte. Systematisches Schlagen wurde während der japanischen Kolonialzeit eingeführt und dann von den Tao übernommen. Für die Anwendung der Körperstrafe müssen schwerwiegende Gründe vorliegen, wie z. B. Diebstahl. Das Schlagen mit dem Stock beginnt mit vier bis fünf 
Jahren. Bei der Häufigkeit und Intensität der Schläge scheint es ein beträchtliches Maß an Varianz zu geben, sodass man nicht von einem klar erkennbaren Muster sprechen kann. Der weit verbreitete Alkoholkonsum führt heute dazu, dass manche Eltern beim Schlagen die Beherrschung verlieren und Kinder misshandeln. Dies widerspricht allerdings dem Ideal von Erziehung, demzufolge Bezugspersonen sich immer unter Kontrolle haben müssen und nie wirklich somozi (wütend) werden dürfen. Meistens übernimmt der Vater die Rolle des Sanktionierenden.

Kinder, die von ihren Bezugspersonen ausgeschimpft oder geschlagen worden sind, richten mitunter ihre Aggressionen auf jüngere und schwächere Personen. Dies geht aus informellen Gesprächen mit Kindern hervor, die angaben, in solchen Situationen ihre jüngeren Geschwister zu schlagen bzw. zu schikanieren.

Eine weitere Form der Sanktionierung besteht im Ignorieren eines sich fehlverhaltenden Kindes. Obwohl diese Form der Disziplinierung bereits vom dritten Lebensjahr an eingeführt wird, erfährt sie erst in der Kindheitsphase ihre eigentliche Ausprägung. Die Tao regulieren generell viel über ihr Blickverhalten. Personen, die man verachtet (ikaoya), schaut man nicht an und redet auch nicht mit ihnen.

\subsection{Erziehungsziel und Emotionsentwicklung}

Das zentrale Erziehungsziel der Tao besteht darin, Kinder für die vielfältigen Gefahren ihres Lebensumfeldes zu sensibilisieren und ihnen eine beständige misstrauische Achtsamkeit zu vermitteln. Kinder müssen lernen, stets auf der Hut zu sein, um drohende Gefahren rechtzeitig zu erkennen. Bedrohungen lauern dabei nicht nur in der naturräumlichen Umgebung, sondern gehen auch von sozialen sowie übernatürlichen Akteuren aus. Eine Person kann sich nur dann vor den vielfältigen Gefahren schützen, wenn sie immer alles im Blick hat und kein Risiko eingeht. Boote können von Meeresströmungen erfasst und aufs offene Meer hinausgetrieben werden; ein falscher Tritt auf dem Korallengestein kann zu Verletzungen führen und wer nicht genau abwägt, an wen er das Fleisch eines geschlachteten Schweins verteilt, ruft unter seinen Mitmenschen Missgunst hervor, die ihm zum Nachteil gereichen kann.

In den ersten beiden Lebensjahren des Kindes leiten die Bezugspersonen durch

Demonstration von Ärger, laute klatschende Geräusche sowie irritierende und ambivalente Praktiken die Sozialisation von maniahey (Angst) ein. Zusammengenommen können diese Strategien als prädispositionales Priming im Sinne von Quinn (2005) verstanden werden. Die Angstdisposition des Kindes wird durch die Beschränkung auf die häusliche Sphäre und die nur sehr langsam erfolgende sukzessive Ausweitung des kindlichen Aktionsradius noch verstärkt, da es seine Umwelt als potentiell gefährlich wahrnimmt.

Kinder müssen in physischer und psychischer Hinsicht abgehärtet werden. Wenn sie sich verletzt haben, dürfen sie nicht weinen und müssen den Schmerz ertragen, da sie ansonsten ausgelacht werden. Bereits zu einem sehr frühen Zeitpunkt im Leben müssen Kinder lernen, alle negativen Emotionen zu regulieren. Nur so kann verhindert werden, dass sie den Attacken der anito-Geister anheimfallen. Zu Abhärtungszwecken werden schon zweijährige Kinder systematisch von ihren Bezugspersonen erschreckt. Obwohl Mütter mit ihren weinenden Kindern Mitleid (ikasi) empfinden, demonstrieren sie in solchen Situationen oft Gleichgültigkeit oder Ärger (somozi), da das vorrangige Sozialisationsziel in einer Verfestigung der Seele besteht. Heranwachsende müssen lernen, ihre Angst zu regulieren und Furcht (masozi) auftreten. Personen, die masozi auftreten, gelten als weniger anfällig für Krankheiten - die wiederum mit den übelwollenden Geistern in einem Zusammenhang stehen. Das Ergebnis dieser emotionalen Sozialisation sind Individuen, die vor nichts Angst haben außer vor der Angst. Unter keinen Umständen dürfen körperliche Symptome von Angst eintreten oder sichtbar werden, wie z. B. ein pochendes Herz oder schneller Atem. Dies wird als ein Indiz dafür verstanden, dass die Seele sich fürchtet (maniahey so pahad) und ist 
gleichbedeutend mit dem Austreten der Seele aus dem Körper oder der Besessenheit von einem übelwollenden anito-Geist. ${ }^{11}$

In einem Experiment wurden 14 Schülern der 5. Klasse gebeten, 20 Emotionswörter, die ihnen gerade durch den Kopf gingen, aufzuschreiben. Die Emotion Angst wurde dabei nur ein einziges Mal genannt. ${ }^{12}$ Bei der Erhebung des Angst-Vokabulars in der Tao-Sprache gaben einige erwachsene Personen an, dass sie keine Angst empfinden würden oder gar noch nie Angst gehabt hätten. Diese Daten deuten an, dass die emotionale Dimension der Angst in der Tao-Gesellschaft ausgeblendet bzw. aufgrund der negativen Bewertung von Angst und Angstdisplays nicht „zugegeben“ wird.

Durch die Induktion von maniahey werden Tao-Kinder für die Unberechenbarkeit ihrer Umwelt sensibilisiert und lernen sich an die sozialen Normen und Verhaltensstandards sowie an die zahlreichen Tabus zu halten. Wir bezeichnen maniahey deshalb als sozialisierende Emotion. Die Sozialisation von Angst überlappt sich mit der Sozialisation von masnek (Scham), die als zweite zentrale sozialisierende Emotion im späteren Verlauf der Ontogenese bei der Vermittlung und Übernahme sozialer Normen eine wichtige Rolle spielt. Die Schamdisposition der Tao-Kinder wird ebenfalls in der oben genannten Priming-Phase vorbereitet. Später kommt es zu einer Intensivierung der Scham-Erfahrung, wenn das Kind bei mangelnder emotionaler Kontrolle von den peers und seinen Bezugspersonen ausgelacht wird. Auch die Praxis des Schikanierens sowie die Erziehungsmethode des Ignorierens tragen zu einer Schamdisposition bei. Aus Platzgründen kann die Schamsozialisation hier nur kurz gestreift werden.

Bedingt durch das folk model der Tao, das zur Verfestigung der zunächst schwachen Kinderseele die Regulation negativer Emotionen in ihrer Gesamtheit vorsieht, ergibt sich eine relative Konturlosigkeit der emotionalen Qualitäten somozi (Ärger, Wut), marahet so onowend (Trauer; negative Emotionen), maniahey (Angst) und masnek (Scham). Diese Konzeptverschmelzung wird auf der linguistischen Ebene durch den übergeordneten Begriff marahet so onowend (negative Emotionalität), der jede der genannten emotionalen Qualitäten umschließt, zum Ausdruck gebracht. Wir fassen deshalb somozi (Ärger, Wut) und marahet so onowned (Trauer; negative Emotionen) als durch die beiden zentralen sozialisierenden Emotionen maniahey (Angst) und masnek (Scham) kulturell überformte Emotionen auf. Kinder als auch Erwachsene gaben bei Befragungen zu Erziehungsmethoden und Erziehungserinnerungen sowie in informellen Gesprächen am häufigsten somozi und marahet so onowned als ihre emotionale Reaktion auf die Evokation von Angst oder Scham an. Die vorläufige Auswertung von über 250 Protokollen systematischer Beobachtung von Emotionsepisoden kann dieses Ergebnis auch auf der Handlungsebene bestätigen. Ein Zusammenhang zwischen Angst und Scham einerseits und den emotionalen Reaktionen Wut und Trauer andererseits wurde schon von vielen Autoren angemerkt (z. B. Lewis 1995; Scheff 1988, 1990; Rosaldo 1984; Robarchek und Robarchek 2005; Casimir 2009; Röttger-Rössler im Druck).

Interessanter weise lassen sich bei der Ausdifferenzierung der Ärger-Emotionen der Tao SubTypen finden, die grob denen der Bara entsprechen. Da Kinder gegenüber älteren Personen der eigenen Verwandtschaftsgruppe Ärger nicht offen zeigen dürfen - wer Widerworte gibt, wird umso härter sanktioniert -, hat sich auch bei den Tao eine Form des Appell-Ärgers herausgebildet. So dürfen Kinder, die beim Essen zu wenig zugewiesen bekommen haben, ihren Unmut (mindok) nur auf eine passive Art und Weise demonstrieren, indem sie ihren zu geringen Anteil beiseite schieben. Die appellierende Wirkung von mindok kann durch das Spitzen des Mundes (ikalangongoy) weiter betont werden. Das Furcht einflößende Auftreten (masozi) v. a. der Männer nach außen hin hält dagegen die Geister in Schach und ist zugleich eine Demonstration von Stärke, die an andere Lineages oder benachbarte Dörfer gerichtet ist. Da ein offenes Ärger-Display generell verpönt ist, artikulieren viele Personen ihren Ärger, 
indem sie seine Verachtungskomponente (ikaoya) betonen und den anderen weder ansehen noch mit ihm reden.

\section{Diskussion}

Die Untersuchungen zu den emotionalisierenden Erziehungspraktiken bei den Bara und Tao und ihren Auswirkungen auf die emotionale Entwicklung der Kinder haben ergeben, dass es zwischen beiden Kulturen sowohl Unterschiede als auch Gemeinsamkeiten gibt. In beiden Kulturen werden Säuglinge zunächst auf proaktive Art und Weise von der Mutter umsorgt. Während sich Bara-Kinder bereits in der Kleinkindphase von der Mutter distanzieren und sich den peers zuzuwenden, setzt diese Entwicklung bei den Tao-Kindern erst mit ca. drei bis vier Jahren ein. Bara-Eltern gehen davon aus, dass ihre Kinder bereits mit vier bis fünf Jahren soziale Normen begreifen und die Sanktion darauf beziehen können; dieser Entwicklungsschritt tritt in der Vorstellung der Tao erst mit 8 bis 12 Jahren endgültig ein. Die Phasenübergänge unterscheiden sich lediglich graduell.

Obwohl in beiden Kulturen Kindesentwicklung als ein kontinuierlicher Prozess begriffen wird, unterscheiden sich die jeweiligen kulturellen Modelle von Entwicklung maßgeblich in der Wahrnehmung der Person-Umwelt-Bezüge: Bei den Tao gilt die Kindesseele als „schwach“ und muss vor den Übel wollenden anito-Geistern bis zum Ende der Kindheit geschützt werden. Diese kulturelle Notwendigkeit führt zu einem grundsätzlich anderen Entwicklungsverlauf als bei den Bara, bei denen Vorstellungen einer derart fragilen Kindesseele fehlen. So ist die von den Bara primär angewandte Erziehungsmethode des Schlagens bei den Tao traditionell verpönt, weil nach indigenen Vorstellungen sich die noch „schwache“ Kindesseele bei Gewaltanwendung fürchtet (maniahey so pahad) und aus dem Körper auszutreten und davonzufliegen droht. Um dies zu vermeiden, müssen die Tao ihre Kinder emotional abhärten, damit ihre Seelen einen festeren Halt im Körper erhalten. Eine Gegenüberstellung der Bara und Tao verdeutlicht, welchen Einfluss die spezifischen folk models auf die jeweils angewendeten Sozialisationspraktiken und die emotionale Entwicklung der Kinder haben können.

Bei den Bara und Tao sind Furcht bzw. Angst (matahotsy bzw. maniahey) die zentralen sozialisierenden Emotionen. Ab dem Kleinkindalter werden Bara- und Tao-Kinder sensibilisiert, sich auf eine furchtsame und respektvolle Art und Weise gegenüber ihren Bezugspersonen zu verhalten. Das entsprechende Verhaltensideal wird bei den Bara durch das Konzept mahitsy und bei den Tao durch die Konzepte mahanang, mamizing und kanig zum Ausdruck gebracht. Die Erziehung bei den Bara vermittelt klare Regeln und erscheint berechenbarer als bei den Tao. Die Furcht auslösende Körperstrafe, die bei den Bara als dominierendes Erziehungsmittel fungiert, kann von den Kindern vermieden werden, wenn sie sich an die bestehenden sozialen Normen halten. Die Bara-Kinder sind somit in der Lage, eindeutige Kausalzusammenhänge für Bestrafungen herzustellen.

Dagegen erscheint der Prozess der Sozialisation bei den Tao vielgestaltiger und diffuser: Einige der frühkindlichen Sozialisationspraktiken (Grimassen ziehen, Verursachen lauter klopfender Geräusche, Aufziehen) haben einen absichtlich irritierenden und ambivalenten Charakter, der Kinder für die Unberechenbarkeit des Lebens sensibilisieren und eine grundsätzliche misstrauische Vorsicht - im Sinne eines existentiellen affektiven Weltbezugs (,existential feeling“, Ratcliffe 2008) - gegenüber der Umwelt sozialisieren soll. Durch die Induktion von Angst (maniahey) werden Tao-Kinder also zunächst auf die vielfältigen Bedrohungen der als grundsätzlich gefährlich konzipierten naturräumlichen, sozialen und übernatürlichen Umwelt vorbereitet und erwerben das im Tao-Kontext erforderliche grundlegende Misstrauen.

Matahotsy bei den Bara und maniahey bei den Tao stellen unseres Erachtens aufgrund ihrer unterschiedlichen kulturellen Einbettung und Sozialisationsfunktionen zwei deutlich 
differente Emotionsqualitäten dar. Da matahotsy die Reaktion auf die Wahrnehmung einer konkreten Bedrohung und somit gerichtet ist, haben wir diese Emotion im vorliegenden Beitrag mit „Furcht“ übersetzt. Das mit der sozialisierenden Emotion maniahey bei den Tao assoziierte generelle Misstrauen lässt i. d. R. jedoch eine klare Gerichtetheit vermissen, so dass wir in diesem Artikel maniahey mit „Angst“ übersetzen. Obwohl in der deutschen Umgangssprache eine Differenzierung zwischen „diffuser Angst“ und „objektbezogener Furcht“ nicht üblich ist, sind diese Begriffszuschreibungen in der Philosophie und Psychologie durchaus verbreitet. Angst leitet sich etymologisch von lateinisch angustia (Enge, Beklemmung, Schwierigkeit) ab und drückt somit auch das Gefühl einer Enge im Brustkorb oder eines Abschnürens der Kehle aus, beides Metaphern, die auf eine veränderte Atmung beim Erleben dieser Emotion verweisen. Genau dies ist der Fall, wenn die Seele nach den Vorstellungen der Tao aus dem Körper austritt.

Die Tatsache, dass bei den Tao neben maniahey (Angst) auch noch masnek (Scham) in der Sozialisation eine dominierende Rolle spielt, mag eine weitere Erklärung für den bei ihnen anzutreffenden diffusen Charakter der Angst sein. Im Gegensatz zu den Bara, bei denen Kinder hauptsächlich die durch die Körpersanktion hervorgerufenen physischen Schmerzen fürchten müssen, kommt bei den Tao noch eine weitere Angst-Dimension hinzu: die Angst vor sozialer Ausgrenzung. Wir sahen, dass bei den Tao Scham (masnek) induzierende ausgrenzende Sozialisationspraktiken wie Verspotten, Verlachen und Ignorieren vorrangig dann zum Einsatz kommen, wenn Kinder die von ihnen ab einem bestimmten Alter sozial erwartete emotionale Kontrolle vermissen lassen, insbesondere, wenn sie ihre Angst noch nicht beherrschen können. Bei den Tao lassen sich also zwei zentrale Entwicklungsschritte voneinander abgrenzen: zunächst wird mittels der sozialisierenden Emotion Angst (maniahey) das als notwendig erachtete existentielle Misstrauen sozialisiert, also eine Angstdisposition herausgebildet, deren Beherrschung und Überwindung den zweiten bedeutenden Entwicklungsschritt darstellt. Hier kommt dann die sozialisierende masnek (Scham) ins Spiel: Kinder, die ihre Emotionen noch nicht zu kontrollieren wissen, die Angst zeigen und weinen oder ihre Wut nicht beherrschen, müssen mit Beschämung, d. h. mit Spott, Schikane und Ausgrenzung rechnen, eine weit verbreitete und effektive Sozialisationspraktik (Quinn 2005; Deonna et al. 2012; Röttger-Rössler im Druck, Röttger-Rössler et al. im Druck).

Ein weiterer Entwicklungsschritt, der hier nur angedeutet werden kann, besteht dann in dem sukzessiven Erwerb von masozi, d. h. der Fähigkeit, aggressiv und Furcht einflößend aufzutreten, was als zentrale Kompetenz in der Auseinandersetzung mit der bedrohlichen Umwelt angesehen wird. In diesem Kontext erscheint auch das Provozieren und „Schikanieren“ (jyasnekan) jüngerer Kinder durch Ältere sinnvoll: Beide Seiten erlernen in den jyasnekan-Interaktionen „Härte“. $\underline{13}$

Wie im ethnographischen Teil zu den Bara dargestellt, empfinden geschlagene Bara-Kinder aber nicht ausschließlich matahotsy (Furcht), sondern auch maseky (Wut) gegenüber dem strafenden Erwachsenen. Die Sanktionierung von aggressivem Verhalten führt zu einer kulturspezifischen Ausdifferenzierung von Wut. Da Wut gegenüber Autoritätspersonen harsch sanktioniert wird, lernen Kinder diese aufgrund von Furcht zu unterdrücken. Sie steht also in einer direkten Relation zu der induzierten Furcht und wird von uns deshalb als sozialisierte Emotion bezeichnet.

Bei den Tao sind die beiden sozialisierenden Emotionen maniahey und masnek zusammen an der Sozialisation von somozi (Ärger, Wut) und marahet so onowned (Trauer, negative Emotionen) beteiligt. Aufgrund der kulturellen Vorstellung eines allmählichen Aushärtungsprozesses der kindlichen Seele müssen im Prozess der Sozialisation negative Emotionen in ihrer Gesamtheit so reguliert werden, dass sie im Display der Personen nicht mehr als solche wahrnehmbar sind. Diese Regulationsleistung wird durch die Angst und Scham induzierenden Sozialisationspraktiken der Tao in die Wege geleitet. 
Sowohl bei den Bara als auch bei den Tao konnte am Beispiel des Ärger-Clusters dargestellt werden, wie sich die indigenen Ärger-Begriffe in Sub-Typen ausdifferenzieren. So gibt es in beiden Gesellschaften eine Form des Appell-Ärgers (mimotso und mihindrotsy bzw. mindok), der sich durch eine Art Schmollen bemerkbar macht und an eine statushöhere Person gerichtet ist. Während aggressive Wut (z. B. may fo) bei den Bara gegen Nichtverwandte aus anderen Lineage-Segmenten gerichtet ist, treten Tao-Männer gegenüber Geistern und rivalisierenden Lineages auf eine Furcht einflößende Art und Weise (masozi) auf.

Die Fokussierung auf emotionalisierende Erziehungsmethoden eignet sich in besonderer Weise, um den Zusammenhang von Kultur und Emotionsentwicklung zu untersuchen. Hierbei halten wir eine holistische Perspektive für unabdingbar. Nur wenn kulturelle Wert- und Überzeugungssysteme, unterschiedliche folk theories über Emotion, Sozialisation und kindliche Entwicklung sowie angemessenes emotionales Ausdrucksverhalten in Relation zu den jeweiligen sozioökonomischen Strukturen und kosmologischen Vorstellungen ausreichend berücksichtigt werden, lässt sich ein tieferes Verständnis kulturspezifischer Emotionscodierungen und emotionaler Kompetenzmodelle erreichen. In diesem Zusammenhang scheint es uns hilfreich, zwischen sozialisierenden und sozialisierten Emotionen zu trennen. Diese analytischen Kategorien erlauben es, die vielgestaltigen biokulturellen sowie sozio-psychologischen Prozesse, die sich in der ontogenetischen Emotionsausbildung verschränken, systematischer zu fassen. Es bedarf allerdings noch zahlreicher weiterer und feiner ziselierter Studien, um diese komplexen Zusammenhänge genauer zu durchdringen.

Literatur

Briggs, J. L. (1982). Living dangerously. The contradictory foundations of value in Canadian Inuit Society. In E. Leacock \& R. Lee (Hrsg.), Politics and history in band societies (S. 109131). Cambridge: Cambridge University Press.

Briggs, J. L. (1998). Inuit morality play. The emotional education of a Three-Year-Old. New Haven: Yale University Press.

Casimir, M. (2009). Honor and dishonor and the quest for emotional equivalents. In B.

Röttger-Rössler \& H. Markowitsch (Hrsg.), Emotions as bio-cultural processes (S. 281-316). New York: Springer.CrossRef

Chang, L., Schwartz, D., Dodge, K., \& McBride-Chang, C. (2003). Harsh parenting in relation to child emotion regulation and aggression. Journal of Family Psychology, 17(4), 598-606.CrossRef

Cole, P. M., Dennis, T. A., Mizuta, I., \& Zahn-Waxler, C. (2002). Self in context. Autonomy and relatedness in Japanese and U.S. mother-preschooler dyads. Child Development, 73(6), 1803-1817.CrossRef

Deonna, J., Rodogno, R., \& Teroni, F. (2011). In defense of shame. The faces of an emotion. Oxford: Oxford University Press.CrossRef

Eisenberg, N., Pidada, S., \& Liew, J. (2001). The relations of regulation and negative emotionality to Indonesian children's social functioning. Child Development, 72(6), 17471763.CrossRef

Friedlmeier, W., \& Trommsdorff, G. (1999). Emotion regulation in early childhood. A crosscultural comparison between German and Japanese toddlers. Journal of Cross-Cultural Psychology, 30(6), 684-711.CrossRef

Fung, H. (1999). Becoming a moral child. The socialization of shame among young Chinese children. Ethos, 27(2), 180-209.CrossRef

Gershoff, E. T. (2002). Corporal punishment by parents and associated child behaviors and experiences: a meta-analytic and theoretical review. Psychological Bulletin, 128(4), 539579.CrossRef

Henrich, J., Heine, S. J., \& Norenzayan, A. (2010). Most people are not WEIRD. Nature, 466, 29. CrossRef 
Holodynski, M. (2004). Die Entwicklung von Emotion und Ausdruck. Vom biologischen zum kulturellen Erbe, ZiF-Mitteilungen, 6, 1-16.

Holodynski, M. (2009). Milestones and mechanisms of emotional development. In B.

Röttger-Rössler \& H. J. Markowitsch (Hrsg.), Emotions as bio-cultural processes (S. 139163). New York: Springer.CrossRef

Holodynski, M., \& Friedlmeier, W. (Hrsg.). (2006). Development of emotions and emotion regulation. New York: Springer.

Huntington, R. (1988). Gender and social structure in Madagascar. Bloomington: Indiana University Press.

Keller, H. (2007). Cultures of infancy. Mahwah: Lawrence Erlbaum.

LeDoux, J. (2002). The synaptic self. How our brains become who we are. Ausgabe: LeDoux, J. (2006). Das Netz der Persönlichkeit. München: DTV.

LeVine, B. B., \& LeVine, R. A. (1966). Nyansongo. A Gusii community in Kenya. New York: Wiley.

LeVine, R. A., \& New, R. S. (Hrsg.). (2008). Anthropology and child development A crosscultural reader. Malden: Wiley-Blackwell.

Levy, R. I. (1984). Emotion, knowing, and culture. In R. A. Shweder \& R. A. LeVine (Hrsg.), Culture theory: Essays on mind, self, and emotion (S. 214-237). Cambridge: Cambridge University Press.

Lewis, M. (1995). Shame. The exposed self. New York: Free Press.

Lieber, E., Fung, H., \& Leung, P. W. L. (2006). Chinese child-rearing beliefs: Key dimensions and contributions to the development of culture-appropriate assessment. Asian Journal of Social Psychology, 9(2), 140-147.CrossRef

Lutz, C. (1983). Parental goals, ethnopsychology, and the development of emotional meaning. Ethos, 11(4), 246-262.CrossRef

Lutz, C. (1988). Unnatural emotions. Everyday sentiment on a Micronesian atoll and their challenge to Western Theory. Chicago: University Of Chicago Press.

Miller, P. J., Fung, H., \& Mintz, J. (1996). Self-construction through narrative practices: A Chinese and American comparison of early socialization. Ethos, 24(2), 237-280.CrossRef Miller, P. J., Wiley, A. R., Fung, H., \& Liang, C.-H. (1997). Personal storytelling as a medium of socialization in Chinese and American families. Child Development, 68(3), 557568.CrossRef

Quinn, N. (2005). Universals of child rearing. Anthropological Theory, 5(4), 477516.CrossRef

Ratcliffe, M. (2008). Feelings of being: Phenomenology, psychiatry and the sense of reality. Oxford: Oxford University Press.

Robarchek, C., \& Robarchek, C. (2005). Waorani grief and the witch-killers rage. Worldview, emotion, and anthroplogical explanation. Ethos, 33(2), 206-230.CrossRef

Rosaldo, R. (1984). Grief and a headhunter's rage: On the cultural force of emotions. In E. Brunner (Hrsg.), Text, play and story: The construction and reconstruction of self and society (S. 178-195). Washington, DC: American Anthropological Association.

Röttger-Rössler, B. (im Druck). In the eyes of the other: Shame and social conformity in the context of Indonesian societies. In B. Sére \& J. Wettlaufer (Hrsg.), Shame between punishment and penance. Micrologus.

Röttger-Rössler, B., Scheidecker, G., Jung, S., \& Holodynski, M. (im Druck). Socializing emotions in childhood: A cross-cultural comparison between the Bara in Madagascar and the Minangkabau in Indonesia. Mind, Culture, and Activity. An International Journal (Special issue on „Psychology of Emotions and Cultural Historical Activity Theory“).

Scheff, T. (1988). Shame and conformity: The deference-emotion system. American Sociology Review, 53(3), 395-406.CrossRef 
Scheff, T. (1990). Socialization of emotions: Pride and shame as causal agents. In T. Kemper (Hrsg.), Research agendas in the sociology of emotions (S. 281-304). Albany: State University of New York Press.

Shipman, K. L., \& Zehman, J. (2001). Socialization of children's emotion regulation in mother-child dyads: A developmental psychopathology perspective. Development and Psychopathology, 13(2), 317-336.CrossRef

Strauss, A., \& Corbin, J. (1990). Basics of qualitative research: Grounded theory procedures and techniques. Thousand Oaks: Sage.

Trommsdorff, G. (2003). Kulturvergleichende Entwicklungspsychologie. In A. Thomas (Hrsg.), Kulturvergleichende Psychologie: Eine Einführung (S. 139-179). Göttingen: Hogrefe.

Fußnoten

1

Dies spiegelt in paradigmatischer Weise der von LeVine und New herausgegebene aktuelle (2008) Sammelband „Anthropology and Child Development“ wider: Unter den 24 Beiträgen dieses Readers, der für die Zusammenarbeit von Ethnologie und Entwicklungspsychologie plädiert, findet sich kein einziger Aufsatz, der Sozialisation und Entwicklung von Emotionen eigens thematisiert.

$\underline{2}$

Vgl. auch die Kritik von Henrich et al. (2010) an der v. a. in der Psychologie vorherrschenden reduktionistischen Beschränkung auf die Untersuchung von Angehörigen euroamerikanischer Mittelschicht.

$\underline{3}$

Dieses Forschungsprojekt wird von der Ethnologin Birgitt Röttger-Rössler (Freie Universität Berlin) in Kooperation mit dem Entwicklungspsychologen Manfred Holodynski (Universität Münster) geleitet. Es wird aus Mitteln der DFG im Rahmen des Exzellenzclusters „Languages of Emotion“ gefördert; s. a.: www.languages-of-emotion.de/de/sozialisation-ontogenese.html.

$\underline{4}$

Biochemische Botenstoffe, die durch emotionale Erregungsprozesse freigesetzt werden, führen zu einer Stärkung der entsprechenden synaptischen Verbindungen, während sie dagegen andere neuronale Vernetzungen abschwächen oder auch ganz auflösen, s. LeDoux 2002, S. 200-234.

5

Unter einer Emotionsepisode verstehen wir eine emotional bedeutsame Interaktion zwischen zwei oder mehreren Akteuren, wobei entweder ein klar erkennbarer Emotionsanlass vorliegt oder zumindest einer der Akteure durch Ausdruckszeichen eine emotionale Erregung sichtbar werden lässt.

$\underline{6}$

Die Feldforschung bei den Bara wurde von Gabriel Scheidecker in insgesamt zwei Aufenthalten von Juni 2009 bis April 2010 sowie von Mai bis August 2011 durchgeführt. $\underline{7}$

Die Darstellung der Körpersanktion basiert v. a. auf Interviews mit 64 Kindern zu ihren Erfahrungen mit der Körpersanktion und mit 22 Erwachsenen zu ihrem Umgang mit 20 verschiedenen Formen von kindlichem Fehlverhalten.

$\underline{8}$

Die Forschungen bei den Tao wurden von Leberecht Funk von Oktober 2010 bis August 2011 durchgeführt.

$\underline{9}$

Für die Versorgung und Erziehung des Kindes ist generell die Mutter zuständig. Wenn sie jedoch Essen zubereitet oder sonst etwas zu erledigen hat, springt der Vater ein und kümmert sich um das Kind. Insgesamt verbringen Väter relativ viel Zeit mit ihrem Nachwuchs. Zu dem 
Kreis der Bezugspersonen gehören häufig auch die Großeltern sowie die Schwestern der Mutter. Aufgrund der Arbeitsmigration nach Taiwan wächst heute ein beträchtlicher Teil der Kinder bei ihren Großeltern auf.

$\underline{10}$

Die Bedeutung von jyasnekan kann am besten mit „,verachten“, „respektlos behandeln“, „provozieren“ oder „schikanieren“ wiedergegeben werden.

$\underline{11}$

Den Tao-Konzeptionen zufolge befindet sich das Selbst in unmittelbarer Gefahr, wenn körperliche Symptome von Angst auftreten, die den potentiellen Austritt der Seele anzeigen. Eine Person, die erkennen lässt, dass sie Angst hat, muss zudem noch soziale Ausgrenzung fürchten. Bekommt z. B. ein Mann beim Fischen Angst und wird dies für die anderen Fischer ersichtlich, so haben sich die anito seiner Seele bemächtigt, was für alle zur Gefahr werden kann, da diese „Geister“ sich dem Boot und der Besatzung anheften könnten. Niemand will den Ängstlichen deshalb weiter an Bord haben. Aus indigener Sicht besteht hier also eine Notwendigkeit zur sozialen Ausgrenzung, was den Druck für die betroffene Person enorm erhöht.

$\underline{12}$

Da Personen unter 35 heute der Muttersprache nicht mehr mächtig sind, wurden die Schüler gebeten, die Emotionswörter auf Chinesisch aufzuschreiben. Die chinesische Entsprechung von maniahey ist ${ }^{3}$ (haipa).

$\underline{13}$

Für Mädchen bzw. Frauen gilt das jedoch nur in eingeschränktem Maße. Es sind hauptsächlich Männer, die masozi-Kompetenzen erwerben müssen, was sich u. a. durch die funktionale Arbeitsteilung der Geschlechter erklären lässt: Es sind die Männer, die am weitesten in die Wildnis vordringen, um aus dem Meer Nahrung zu beschaffen oder in der unwegsamen Bergwelt Holz für die Boote zu schlagen. Ihr Furcht erregendes Auftreten ist somit eine zentrale Kompetenz, um in der feindlichen und bedrohlichen Umwelt bestehen zu können. Durch die Demonstration ihrer Kraft und Aggression sind sie zudem in der Lage, rivalisierende Lineages in ihre Schranken zu verweisen. Die Genderspezifik der Emotionsentwicklung kann jedoch hier nicht näher behandelt werden. 